\title{
Prevalencia de dolor crónico en personas adultas en Costa Rica, enero-2020 a marzo-2021.
}

\author{
Prevalence of chronic pain in adults in Costa Rica, Jan-2020 to March-2021.
}

\begin{abstract}
Filiación:
${ }^{1}$ Universidad Santa Paula, Curridabat, San José, Costa Rica. 2Instituto Costarricense de Investigación y Enseñanza en Nutrición y Salud, INCIENSA, Cartago, Costa Rica.

Correspondencia: $\square$ Carlos Fernando Acuña Aguilar, correo electrónico:

cacuna@uspsantapaula.com

- - - - - - - - - -

Forma de citar: AcuñaAguilar C, Fernández-Cordero A, Benavides-Lara A. Prevalencia de dolor crónico en personas adultas en Costa Rica. Rev Ter. 2022;16(1): 50-66.
\end{abstract}

Financiamiento:

ABBOTT Centroamérica y EI Caribe.

Conflictos de Interés:

Ninguno

Abreviaturas:

ACHED, Asociación Chilena para el Estudio del Dolor; AINES, antinflamatorios no esteroideos; AVD, actividades de la vida diaria; CEC-USP, Comité Ético Científico de la Universidad Santa Paula; CONIS, Consejo Nacional de Investigación en

Salud; $\quad$ DOLCA, estudio epidemiológico del dolor crónico en Caldas, Colombia; EEUU, Estados Unidos; FEDELAT, Federación Latinoamericana de Asociaciones para el Estudio del Dolor; IAS, International Association for The Study of Pain (Asociación Internacional para el estudio del Dolor); ICE, Instituto Costarricense de Electricidad; INEC, Instituto Nacional de Estadística y Censos; OMS, Organización Mundial de la Salud.

Fecha de envío: 10 de junio del 2021.

Fecha de aceptación: 28 de junio del 2021.

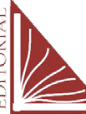

Carlos Acuña-Aguilar ${ }^{1} \square$, Ariel Fernández-Cordero ${ }^{1}$, Adriana Benavides-Lara $^{1,2}$

\section{Resumen}

Introducción: el objetivo del estudio fue determinar el comportamiento epidemiológico y la atención de las personas adultas que cursan con dolor crónico en Costa Rica. Metodología: estudio observacional transversal mediante una encuesta telefónica realizada a personas mayores de 19 años, residentes en Costa Rica por al menos tres meses. Se estimó una muestra aleatoria con representatividad nacional, ponderada según los 82 cantones del país. Se determinó la prevalencia de dolor crónico con su respectivo IC95\% para el país y cada provincia. Resultados: se realizaron entrevistas a 375 personas, con una media de edad de 57,4 años (DE 17,4); 73,1\% mujeres. Ciento ocho personas reportaron dolor crónico para una prevalencia nacional de 28,8\% (IC95\% 24,3-33,7), con una media de edad significativamente mayor que la población entrevistada (62,9 años; DE 14,2). No se encontraron diferencias por provincia. La media de duración del dolor fue 61 meses (DE: 104,7; rango 36-756 meses). La artrosis como causa única se presentó en el $65,6 \%$ de los casos. El miembro inferior fue la localización más frecuente $(53,7 \%)$. Para la intensidad del dolor (escala numérica del 0 a 10): 69,4\% puntuó igual o superior a 5, y 29,6\% entre 8-10. Según su frecuencia, $54 \%$ presentaron dolor a diario; la mayoría lo describieron como entumecimiento $(40,7 \%)$. Aproximadamente $83,3 \%$ utilizaron medidas farmacológicas, principalmente antiinflamatorios no esteroideos (76,9\%); $64 \%$ de estos medicamentos fueron prescritos por un médico, 30\% se auto medicaron o siguieron consejos (publicidad o amigos). La esfera de vida diaria más afectada fue la laboral $(70,4 \%)$, seguida de alteraciones en el sueño $(59,3 \%)$. Conclusión: el dolor crónico en las personas adultas de Costa Rica es un problema de salud pública, con una prevalencia similar a la reportada en Latinoamérica, que aumenta significativamente con la edad. La mayoría de las personas que lo padecen se ven afectadas diariamente por periodos prolongados. Se destaca la baja utilización de opioides, un elevado consumo de AINES y automedicación.

Palabras claves: prevalencia, dolor crónico, epidemiología, América Latina. 


\section{Abstract}

Introduction: The aim of the study was to determine the epidemiological behavior and care of adults with chronicle pain. Methodology: A cross-sectional observational study was conducted through a telephone survey (home telephone), to people over 19 years , resident in Costa Rica for at least three months, estimated a random sample with national representativeness, weighted according to the 82 cantons of the country. The prevalence of chronic pain was determined with its respective $\mathrm{Cl} 95 \%$ for the country and each province. Results: interviews were conducted to 375 people in an age range of 57.4 years (SD 17.4), $73.1 \%$ women. One hundred and eight people reported chronic pain for a national prevalence of $28.8 \%$ (95\% Cl 24.3-33.7), with a mean age significantly higher than the interviewed population (62.9 years; SD 14.2). None difference were found by province. The mean duration of pain was 61 months (SD: 104.7; range 36-756 months). Osteoarthritis, as a single cause, occurred in $65.6 \%$ of cases. The lower limb was the most frequent location $53.70 \%$. For the intensity of pain (numerical scale from 0 to 10 ): $69.4 \%$ scored equal to or greater than $5,29.6 \%$ was placed on the scale between 8 to 10. According to their frequency, $54 \%$ had pain on a daily basis; most described it as numbness (40.74\%). Eighty-three percent used pharmacological measures, mainly no steroidal, anti-inflammatory drugs were used by a $76.9 \% ; 64 \%$ of these was prescribed by a professional in medicine, $30 \%$ self-medicated or follow advertising advice or friend recommendation. The working area was the most affected sphere of daily life $(70.37 \%)$, followed by sleep disorders (59.3\%). Conclusion: chronicle pain in adults in Costa Rica is a public health problem, with prevalence similar to the one reported in Latin America, that increases significantly with age. Most people, who suffer from it, are affected daily for prolonged period. It highlights the low use of opioid, a high consumption of NSAIDS and self-medication.

Keywords: prevalence, chronicle pain, epidemiology, Latin America.

\section{Introducción}

El dolor ha sido definido por la Asociación Internacional para el Estudio del Dolor (IASP, siglas en inglés) como: "una experiencia sensorial y emocional desagradable asociado a un daño tisular, real o potencial, descrito en términos de ese daño"1. El dolor físico señala una sensación molesta, aflictiva que se genera en el cuerpo y trasciende todas las dimensiones del ser humano. Es clasificado según su localización, duración, intensidad, características, irradiación, factores atenuantes o agravantes, frecuencia, síntomas asociados, entre otros ${ }^{2}$. Por su duración se clasifica en dolor agudo y crónico; el agudo se encuentra relacionado con la magnitud del daño tisular y se constituye en una función biológica, útil y una herramienta protectora del ser humano. El crónico es definido como aquel dolor que persiste más allá del tiempo de la recuperación del daño, o aquel que perdura o recurre más allá de tres meses. El dolor crónico no cumple una función biológica útil y se convierte en una fuente que puede generar sufrimiento, discapacidad $y$ afectación en la calidad de vida de las personas que lo padecen. En ocasiones la cronicidad puede ser por meses e inclusive años, la mayoría de las veces pasa a ser una condición patológica por sí 
misma e independiente de la enfermedad o daño tisular primario que lo ocasionó. Esto ha hecho que sea considerado como una enfermedad por derecho propio $^{3}$.

El dolor crónico es una condición multifactorial que afecta todas las dimensiones del ser humano y puede generar necesidades físicas, psicológicas, sociales e inclusive psico-existenciales, como: afectación del nivel de funcionalidad, trastornos neuropsicológicos (depresión y/o ansiedad), problemas de adaptación, cambio en los hábitos del sueño, catastrofización ante el dolor, relaciones interpersonales alteradas, aislamiento, afectación a nivel laboral (ausentismo o disminución de la productividad), discapacidad e invalide $z^{4,5}$.

En la actualidad, es considerado un problema de salud pública debido a su alta prevalencia, carga de enfermedad y costo económico, afecta entre un 20 a 50\% de la población general y es la causa líder de discapacidad a nivel mundial, es más prevalente en países más pobres y con limitaciones para la educación ${ }^{3}$.

El dolor crónico es un problema que afecta a 1,5 billones de personas a nivel mundial ${ }^{5}$. Según Covarrubias Gómez et $\mathrm{al}^{7}$, la prevalencia de dolor crónico en países desarrollados es del $20 \%$ y el promedio mundial según la $\mathrm{IASP}^{8}$ es del $25 \%$. Asimismo, el $20 \%$ de las personas adultas sufren de dolor y el $10 \%$ es diagnosticado como dolor crónico cada año ${ }^{9}$.

En el 2006 en el estudio "Pain in Europe" realizado en 15 países europeos más Israel, en 46 394 encuestas telefónicas efectuadas a personas adultas, se determinó un promedio de dolor crónico del 19\%. En países como Noruega, Polonia e Italia, este ascendió hasta $25 \%$, siendo España el país con el promedio más bajo de un $11 \%{ }^{10}$.
En Costa Rica no existen estudios acerca de la prevalencia del dolor crónico, sin embargo, se reconoce que se trata de un problema de salud pública, por la alta frecuencia de consultas médicas y de la prescripción de medicamentos analgésicos a nivel nacional. Con el afán de darle continuidad a la iniciativa del Comité de Epidemiología de la Federación Latinoamericana de Asociaciones para el Estudio del Dolor (FEDELAT), así como el interés de la IASP, de fomentar la investigación de dolor en el mundo, lo cual está consagrado en sus estatutos desde el momento de su creación, surge la necesidad de realizar este estudio para conocer la prevalencia y principales características del dolor crónico en adultos de Costa Rica.

El escaso conocimiento de la realidad costarricense en materia de dolor crónico, así como el de algunas enfermedades crónicas y degenerativas lleva a la carencia de políticas sanitarias claras para la prevención y el tratamiento oportuno de estas enfermedades, que contribuyan en evitar la progresión y consecuencias de estas.

\section{Materiales y métodos}

Se realizó un estudio observacional transversal por medio de una encuesta telefónica realizada entre enero 2020 a marzo 2021, a personas mayores de 19 años de ambos sexos, residentes en Costa Rica por al menos tres meses, que contaran con un número telefónico domiciliar o fijo registrado en el directorio telefónico del Instituto Costarricense de Electricidad (ICE) de Costa Rica del 2018, que estuvieran en capacidad de resolver la encuesta por vía telefónica y que dieran su consentimiento informado. Este estudio contó con la aprobación del Comité Ético Científico de la Universidad Santa 
Paula (CEC-USP) y fue debidamente registrado en el Consejo Nacional de Investigación en Salud (CONIS), en estricto apego a la ley de investigación biomédica costarricense. No se reportó ningún tipo de evento adverso en los participantes del estudio.

Como instrumento de encuesta se utilizó un Cuestionario de Dolor, elaborado por el Comité de Epidemiología de la FEDELAT ${ }^{11}$, que ha sido aplicado y validado para determinar la prevalencia del dolor crónico en Colombia, México, Chile y Brasil.

Utilizando como marco muestral el directorio telefónico del ICE actualizado para el 2018, se elaboró una lista de números telefónicos en orden ascendente (para eliminar el orden alfabético de los números del directorio) segregados por cantón. A cada número telefónico de 8 dígitos, se le asignó un número consecutivo; con respecto a este último consecutivo se realizó un muestreo aleatorio (tabla de números aleatorios, software Epidat versión 4.2), no estratificado, ponderado según cantón de acuerdo con la población adulta (de 19 años en adelante) de cada uno de los 82 cantones del país. De acuerdo con la prevalencia estimada para Latinoamérica se obtuvo un número de muestra de 369 personas como mínimo para estimar la prevalencia con un $95 \%$ de confianza. El proceso de muestreo tuvo que correrse en tres ocasiones dada la frecuente negativa de las personas a atender preguntas telefónicas, así como una gran cantidad de números telefónicos que no estaban habilitados durante el proceso de encuesta.

Se determinó la prevalencia de dolor crónico con su respectivo IC95\% para el país y para cada provincia, tomando como numerador el número de personas que cumplan con la definición de dolor crónico y como denominador el total de personas entrevistadas que contestaron la encuesta. Se realizó un análisis descriptivo por medio de medidas de tendencia central (media, desviaciones estándar, rango) para variables numéricas tales como edad, tiempo de evolución del dolor, duración del dolor, y para variables categóricas se realizaron distribuciones de frecuencias con sus respectivos intervalos de confianza al 95\% (IC95\%), las proporciones se compararon según la prueba de Chi-cuadrado. La significancia estadística se determinó mediante un $\alpha \leq 0,05$. Para el análisis estadístico de la información se utilizó el programa Epi Info 7 versión 7.2.4.0.

\section{Resultados}

Se entrevistaron un total de personas adultas de todas las provincias del país con una media de edad de 57,4 años (DE 17,4, Mediana 72 años). La distribución según de los entrevistados fue 73,1\% mujeres y $26,9 \%$ hombres.

Del total de personas entrevistadas 267 no presentaron dolor crónico, y 108 personas dijeron tener dolor crónico (superior o igual a tres meses de duración) para una prevalencia nacional de dolor crónico de 28,8\% (IC95\% 24,3-33,7). Las distribuciones de las personas con y sin dolor según sexo, grupo de edad y provincia de procedencia se detallan en la Tabla 1.

En la sub-muestra de personas con dolor hubo significativamente mayor número de mujeres, para una relación de 2,7 mujeres por cada hombre con dolor crónico. No obstante, no se encontraron diferencias significativas en la distribución por sexo entre las personas con y sin dolor, así que es probable que esta relación mujer/hombre esté influenciada por la mayor cantidad de mujeres que contestaron la encuesta. 
Tabla1. Distribución de personas entrevistadas por sexo, grupo de edad y provincia, según presencia o ausencia de dolor crónico. Costa Rica 2020

\begin{tabular}{|c|c|c|c|c|}
\hline \multirow[t]{2}{*}{ Variables } & \multicolumn{2}{|c|}{ Dolor crónico } & \multirow[t]{2}{*}{ Total } & \multirow[t]{2}{*}{ Valor $p$} \\
\hline & Con dolor (n:108) & Sin dolor (n:267) & & \\
\hline GENERO & & & & 0,1 \\
\hline Femenino & 85 & 189 & 274 & \\
\hline \% según filas (variables) & $31,0 \%$ & $69,0 \%$ & $100,0 \%$ & \\
\hline \% por columnas (dolor crónico si/no) & $78,7 \%$ & $70,8 \%$ & $73,1 \%$ & \\
\hline Masculino & 23 & 78 & 101 & \\
\hline \% según filas (variables) & $22,8 \%$ & $77,2 \%$ & $100,0 \%$ & \\
\hline \% por columnas (dolor crónico si/no) & $21,3 \%$ & $29,2 \%$ & $26,9 \%$ & \\
\hline GRUPO DE EDAD * & & & & $<0,01$ \\
\hline $19-49$ & 19 & 92 & 111 & \\
\hline \% según filas (variables) & $17,1 \%$ & $82,9 \%$ & $100,0 \%$ & \\
\hline \% por columnas (dolor crónico si/no) & $17,6 \%$ & $35,1 \%$ & $30,0 \%$ & \\
\hline $50-64$ & 36 & 84 & 120 & \\
\hline \% según filas (variables) & $30,0 \%$ & $70,0 \%$ & $100,0 \%$ & \\
\hline \% por columnas (dolor crónico si/no) & $33,3 \%$ & $32,1 \%$ & $32,4 \%$ & \\
\hline Mayor o igual 65 & 53 & 86 & 139 & \\
\hline \% según filas (variables) & $38,1 \%$ & $61,9 \%$ & $100,0 \%$ & \\
\hline \% por columnas (dolor crónico si/no) & $49,1 \%$ & $32,8 \%$ & $37,6 \%$ & \\
\hline PROVINCIA & & & & 0,2 \\
\hline Alajuela & 29 & 45 & 74 & \\
\hline \% según filas (variables) & $39,2 \%$ & $60,9 \%$ & $100,0 \%$ & \\
\hline \% por columnas (dolor crónico si/no) & $26,9 \%$ & $16,9 \%$ & $19,8 \%$ & \\
\hline Cartago & 15 & 32 & 47 & \\
\hline \% según filas (variables) & $31,9 \%$ & $68,1 \%$ & $100,0 \%$ & \\
\hline \% por columnas (dolor crónico si/no) & $13,9 \%$ & $12,0 \%$ & $12,5 \%$ & \\
\hline Guanacaste & 5 & 18 & 23 & \\
\hline \% según filas (variables) & $21,7 \%$ & $78,3 \%$ & $100,0 \%$ & \\
\hline \% por columnas (dolor crónico si/no) & $4,6 \%$ & $6,7 \%$ & $6,1 \%$ & \\
\hline Heredia & 8 & 36 & 44 & \\
\hline \% según filas (variables) & $18,2 \%$ & $81,8 \%$ & $100,0 \%$ & \\
\hline \% por columnas (dolor crónico si/no) & $7,4 \%$ & $13,5 \%$ & $11,7 \%$ & \\
\hline Limón & 15 & 35 & 50 & \\
\hline \% según filas (variables) & $30,0 \%$ & $70,0 \%$ & $100,0 \%$ & \\
\hline \% por columnas (dolor crónico si/no) & $13,9 \%$ & $13,1 \%$ & $13,3 \%$ & \\
\hline Puntarenas & 5 & 24 & 28 & \\
\hline \% según filas (variables) & $17,9 \%$ & $85,7 \%$ & $100,0 \%$ & \\
\hline \% por columnas (dolor crónico si/no) & $4,6 \%$ & $8,6 \%$ & $7,5 \%$ & \\
\hline San José & 31 & 77 & 108 & \\
\hline \% según filas (variables) & $28,7 \%$ & $71,3 \%$ & $100,0 \%$ & \\
\hline \% por columnas (dolor crónico si/no) & $28,7 \%$ & $28,8 \%$ & $28,8 \%$ & \\
\hline
\end{tabular}

* 5 personas del grupo sin dolor no quisieron decir su edad, solo que eran más de 19 años.

Fuente: elaboración de los autores 
Al analizar las sub-muestras (con dolor y sin dolor) la distribución por edad si mostró diferencias significativas, siendo que las personas con dolor crónico presentaron una media de edad significativamente más alta de 62,9 años (DE 14,2; Mediana 72,5; Rango 19-91), que las personas sin dolor que fue de 55 años (DE 17,7; Mediana 68;
Rango 19-99). La Figura 1 muestra la distribución por edad según presentan o no presentan dolor, donde es evidente la diferencia de distribución porcentual entre ambos grupos, siendo que en las personas con dolor crónico el mayor porcentaje de personas se distribuyen en edades de 60 años y más.

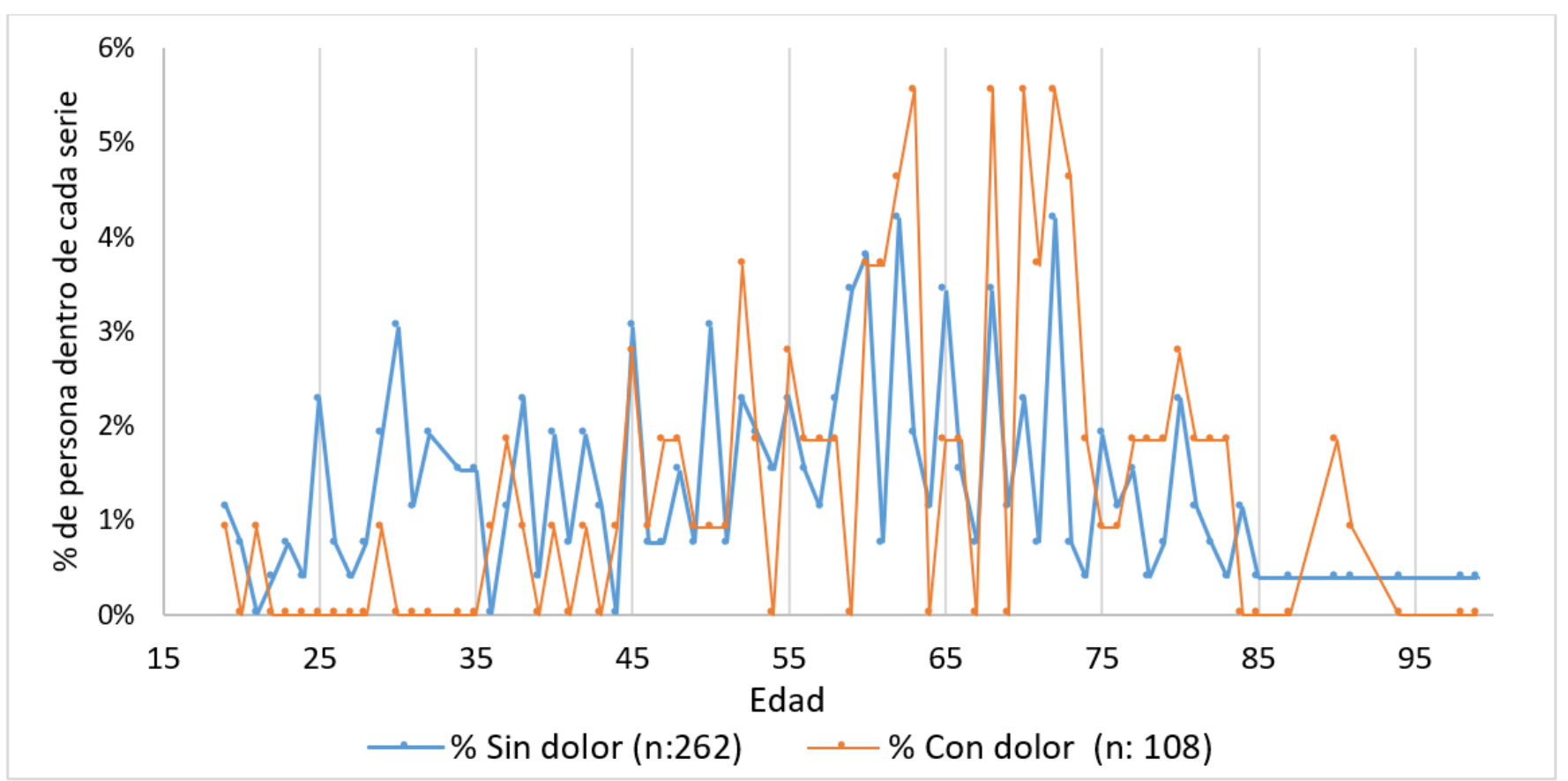

Figura 1. Distribución porcentual por edad de las personas según presencia o ausencia de dolor crónico. Costa Rica enero 2020- marzo 2021

Fuente: elaboración de los autores con base en datos de entrevista.

Según provincia, la prevalencia de dolor crónico varió entre $17,9 \%$ y 39,2\% correspondientes a las provincias de Puntarenas y Alajuela respectivamente. Sin embargo, comparando los IC95\% de las prevalencias por provincias estos se traslapan con el IC95\% a nivel nacional, lo que significa que no se observaron diferencias estadísticamente significativas con respecto a la prevalencia nacional que fue de $28,8 \%$. Cabe aclarar que la proporción de personas entrevistadas según provincia guarda relación con la densidad de población adulta de cada una de ellas.

\section{Descripción de sub-muestra de personas con dolor crónico (n: 108)}

\section{Tiempo con dolor}

La media según el tiempo con dolor crónico 
en meses fue de 61 meses (DE: 104,7; rango 36756, moda 60 meses), que equivale a 5 años y un mes, el rango de meses con dolor es amplio. El porcentaje mayor $(71,3 \%)$ se presentó en el grupo de participantes que tuvieron dolor entre 12 a 120 meses. El participante con mayor tiempo con dolor fue un hombre que fue afectado con poliomielitis en su niñez, con consecuencias de dolor crónico por más de 720 meses (60 años).

\section{Causas de dolor}

De las 108 personas entrevistadas con dolor crónico, cuatro de ellos desconocían su causa (3,7\%) porque nunca recibieron un diagnóstico claro. De los 104 pacientes que decían conocer la causa de su dolor, 31,7\% (IC95\% 23,0-41,6\%) presentó más de una causa. La artrosis, la lumbalgia y la fibromialgia fueron las causas más frecuentes de dolor crónico, siendo las menos frecuentes el cáncer, el herpes zoster, la artritis y los trastornos digestivos (gastritis, colitis). El estrés, los problemas neurológicos, la fibromialgia y la lumbalgia arrojaron probabilidades porcentuales significativas de presentarse en combinación con otras causas y no como causa única; todas las personas entrevistadas que señalaron el estrés como causa, lo anotaron como parte de una combinación de estas, mientras que la artrosis, causa más frecuente, se presentó en un $65,6 \%$ como única causa. La Tabla 2 muestra con detalle la distribución de las causas de dolor crónico descrita por el entrevistado y su probabilidad de presentarse en combinación con otras causas; así pues, una misma persona podría referir más de una de las causas descritas en la tabla.

\section{Localización del dolor}

La distribución según localización del dolor se presenta en la Tabla 3, el miembro inferior, seguido del miembro superior y la espalda son los sitios más frecuentes.

\section{Características del dolor}

Para medir la intensidad del dolor se tomó como parámetro una escala numérica del 0 al 10 , donde 0 es la ausencia de dolor y 10 el peor dolor que se pueda imaginar. Ciento siete de los 108 entrevistados con dolor crónico contestaron la pregunta. La Figura 2 muestra la distribución de las personas que padecían dolor crónico de acuerdo con la escala numérica del dolor; la mayoría de las personas entrevistadas (n: $56 ; 52,3 \%$ ) se ubicaron en intensidad entre 5 y 7, 30\% (32 de 107) se ubicaron en la intensidad igual o superior a 8 .

En lo que respecta a la frecuencia del dolor (Figura $3 A$ ), la mayoría de personas (54,6\%; IC95\% 44,8$64,2)$ presentaban el dolor a diario. En relación con la duración del dolor (Figura 3B), un alto porcentaje de las personas $(33,3 \%$, IC95\% 24,5-43,1) refirieron que el dolor es constante ("todo el tiempo" según refirieron) y sólo 14 (13,0\%; IC95 7,3-20,8) lo describieron como un dolor momentáneo. Una persona no respondió acerca de características de duración e intensidad.

Las características particulares asociadas al dolor crónico en este estudio se presentan en la Tabla 4. El entumecimiento en la zona dolorosa, aumento del dolor con la presión y sensación de pinchazos y agujas son las características más frecuentemente asociadas al dolor crónico. 
Tabla 2. Distribución de causas de dolor crónico y probabilidad de que estas causas se presenten en combinación con otras. Costa Rica enero 2020- marzo 2021

\begin{tabular}{lccccll}
\hline Causa & $\mathbf{n}$ & $\%$ & \multicolumn{2}{c}{ IC 95\% } & $\begin{array}{l}\text { \% combinación } \\
\text { con otras causas }\end{array}$ & \multicolumn{2}{l}{ Valor $\mathbf{p}^{*}$} \\
\hline Cancer & 1 & $0,9 \%$ & & & & - \\
Herpes & 2 & $1,9 \%$ & $0,2 \%$ & $6,5 \%$ & $50,0 \%$ & 0,8 \\
Artritis & 2 & $1,9 \%$ & $0,2 \%$ & $6,5 \%$ & $50,0 \%$ & 0,8 \\
Trastorno digestivo & 4 & $3,7 \%$ & $1,0 \%$ & $9,2 \%$ & $100,0 \%$ & 0,1 \\
Post traumatico (caídas) & 4 & $3,7 \%$ & $1,0 \%$ & $9,2 \%$ & $0 \%$ & 0,1 \\
Diabetes & 5 & $4,6 \%$ & $1,5 \%$ & $10,5 \%$ & $75,0 \%$ & 0,1 \\
Estrés & 5 & $4,6 \%$ & $1,5 \%$ & $10,5 \%$ & $100,0 \%$ & $<0,01$ \\
Osteoporosis & 7 & $6,5 \%$ & $2,7 \%$ & $12,9 \%$ & $57,1 \%$ & 0,3 \\
Migraña & 8 & $7,4 \%$ & $3,3 \%$ & $14,1 \%$ & $0 \%$ & $<0,01$ \\
Problema circulatorio & 8 & $7,4 \%$ & $3,3 \%$ & $14,1 \%$ & $50,0 \%$ & 0,4 \\
Problema neurológico & 9 & $8,3 \%$ & $3,9 \%$ & $15,2 \%$ & $54,0 \%$ & $<0,05$ \\
Fibromialgia & 14 & $13,0 \%$ & $7,3 \%$ & $20,8 \%$ & $57,0 \%$ & $<0,05$ \\
Lumbalgia & 24 & $22,2 \%$ & $14,8 \%$ & $31,2 \%$ & $54,2 \%$ & $<0,05$ \\
Artrosis & 32 & $29,6 \%$ & $21,2 \%$ & $39,2 \%$ & $34,4 \%$ & 0,4 \\
Otros & 9 & $8,3 \%$ & $3,9 \%$ & $15,2 \%$ & $0 \%$ & $<0,01$ \\
\hline
\end{tabular}

Nota: Se detallan los casos de pacientes que presentaban una causa específica, ya sea aislada o en combinación con otras causas. Así pues, un mismo paciente podía citar varias causas de su dolor crónico.

* valor de $p$ de la probabilidad de la causa específica de presentarse combinada con otras causas.

1: esta categoría incluye otras causas que se presentaron aisladas diferentes a las mencionadas en la tabla entre ellas, hernia hiatal, Esclerosis múltiple, Lupus Eritematoso sistémico, Enfermedad de Hashimoto, Poliomelitis, dolor inespecífico de miembro inferior, gonalgia inespecífica, exceso de ácido úrico, problema genético no especificado, tumor benigno en región poplítea y cervicalgia inespecífica y dolores neuromusculares de causa no especificada.

Fuente: elaboración de los autores

Tabla 3. Frecuencia de personas que presentaron dolor crónico según la/las localizaciones del dolor que manifestaron los encuestados. Costa Rica, enero 2020- marzo 2021

\begin{tabular}{lcccc}
\hline Localización & $\mathbf{n}$ & $\%$ & \multicolumn{2}{c}{ IC95\% } \\
\hline Cabeza y cuello & 19 & $17,59 \%$ & $10,94 \%$ & $26,10 \%$ \\
Miembro superior & 48 & $44,44 \%$ & $34,88 \%$ & $54,32 \%$ \\
Torax & 4 & $3,70 \%$ & $1,02 \%$ & $9,21 \%$ \\
Abdomen & 2 & $1,85 \%$ & $0,23 \%$ & $6,53 \%$ \\
Espalda (lumbo/sacro) & 42 & $38,89 \%$ & $29,66 \%$ & $48,75 \%$ \\
Cadera & 20 & $18,52 \%$ & $11,69 \%$ & $27,14 \%$ \\
Miembro inferior & 58 & $53,70 \%$ & $43,85 \%$ & $63,35 \%$ \\
Otros & 2 & $1,85 \%$ & $0,23 \%$ & $6,53 \%$ \\
\hline
\end{tabular}

Nota: $27,1 \%$ de los entrevistados tenían dolor en más de una localización

Fuente: elaboración de los autores 


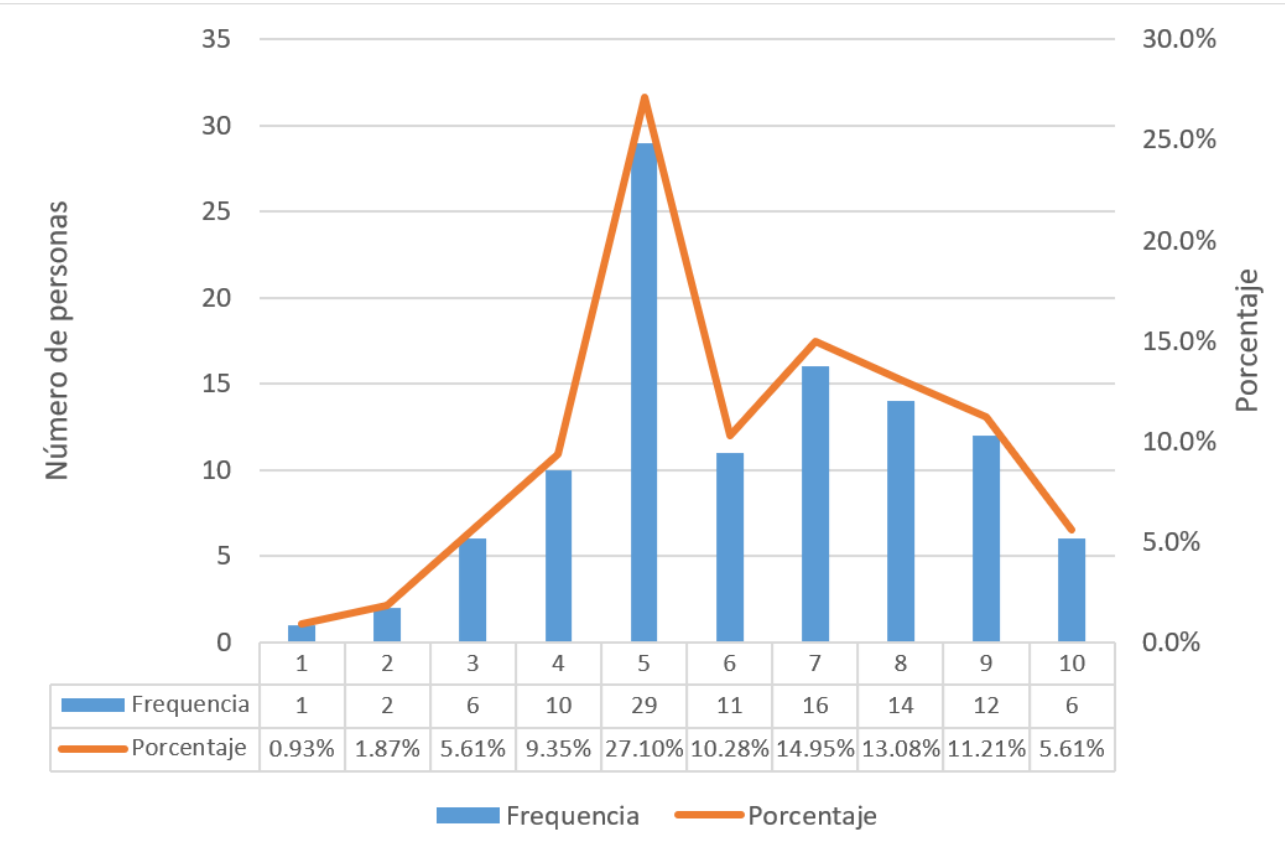

Figura 2. Distribución de las personas con dolor crónico según escala numérica de intensidad de dolor. Costa Rica enero 2020- marzo 2021

Fuente: elaboración de los autores con base en datos de entrevista.

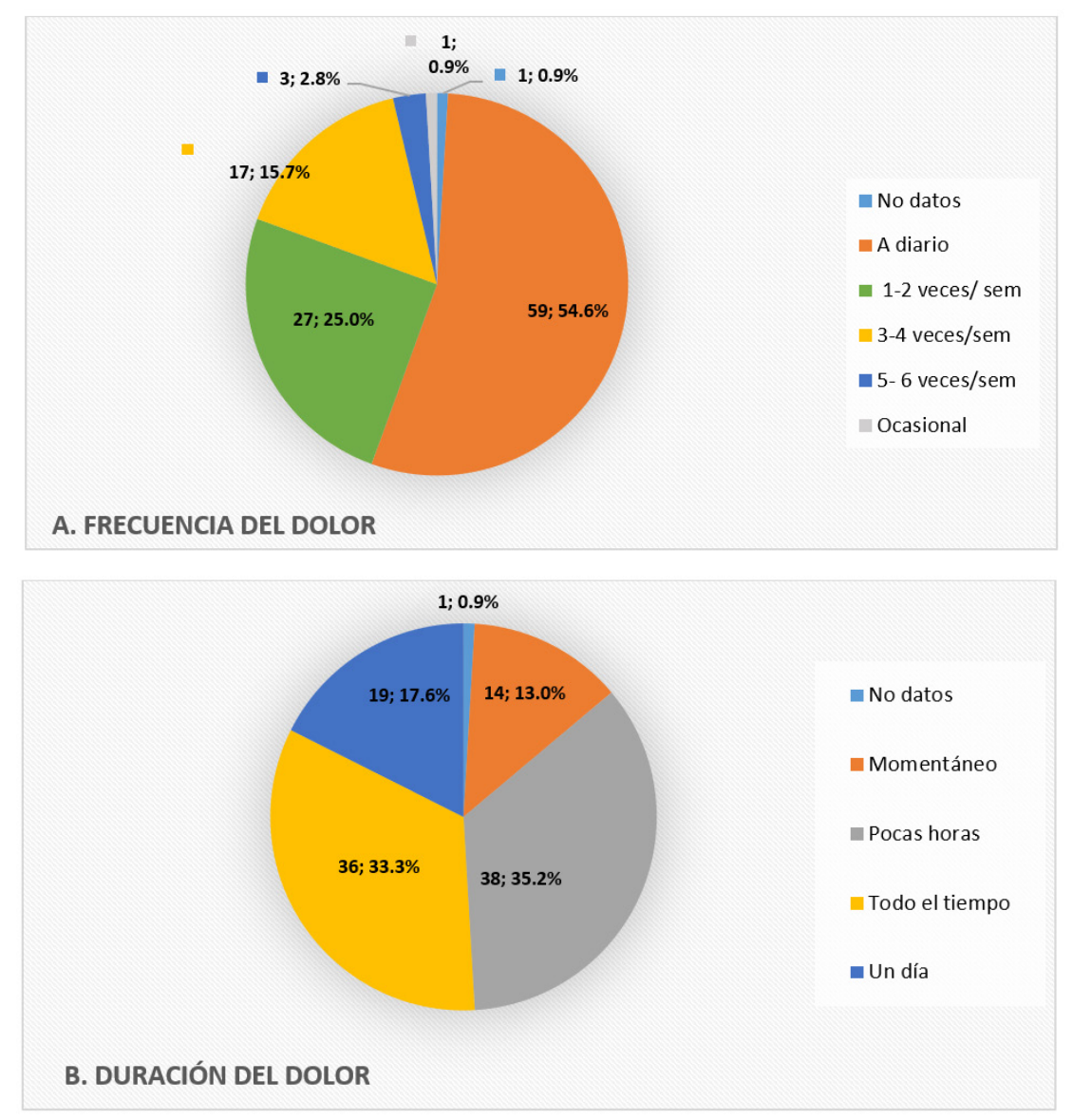

Figura 3. Distribución de las personas (n;\%) con dolor crónico según frecuencia del dolor (A) e intensidad del dolor (B). Costa Rica, enero 2020- marzo 2021

Fuente: elaboración de los autores con base en datos de entrevista. 
Tabla 4. Distribución de personas según las características sensoriales del dolor crónico. Costa Rica, enero-2020 a marzo-2021

\begin{tabular}{lcccc}
\hline Característica & Frecuencia & $\%$ & \multicolumn{2}{c}{ IC95\% } \\
\hline Entumecimiento & 44 & $40,7 \%$ & $31,4 \%$ & $50,6 \%$ \\
Aumenta con la presión & 43 & $39,8 \%$ & $30,5 \%$ & $49,7 \%$ \\
Sensación de pinchazos & 25 & $23,2 \%$ & $15,6 \%$ & $32,3 \%$ \\
Sensación de hormigueo & 22 & $20,4 \%$ & $13,2 \%$ & $29,2 \%$ \\
Sensación de quemadura & 21 & $19,4 \%$ & $12,5 \%$ & $28,2 \%$ \\
Sensación de electricidad & 17 & $15,7 \%$ & $9,5 \%$ & $24,0 \%$ \\
Sensación de agujas & 17 & $15,7 \%$ & $9,5 \%$ & $24,0 \%$ \\
Disminución de la sensibilidad & 10 & $9,3 \%$ & $4,5 \%$ & $16,4 \%$ \\
Aumenta con roce suave & 8 & $7,4 \%$ & $3,3 \%$ & $14,1 \%$ \\
Asocia picazón & 8 & $7,4 \%$ & $3,3 \%$ & $14,1 \%$ \\
Sensación de frío & 5 & $4,6 \%$ & $1,5 \%$ & $10,5 \%$ \\
\hline
\end{tabular}

Nota: 2 pacientes de 108 (1,85\%) no contestaron las características del dolor. Una misma persona puede presentar dolor con varias características sensoriales.

Fuente: elaboración de los autores con base en datos de entrevista

\section{Tipo de tratamiento o terapia}

De 108 personas con dolor crónico, 13 personas $(12,0 \%)$, utilizaron más de un tipo de tratamiento, entre los cuales estan: medicamentos (90; $83,3 \%)$, terapia física $(8 ; 7,4 \%)$ terapias alternativas $(7 ; 6,5 \%)$ y bloqueos nerviosos (2; $1,9 \%)$, mientras que 3 personas $(2,7 \%)$ no utilizaron ningún tipo de tratamiento. Así pues, el tratamiento farmacológico es el más utilizado; de las personas que utilizaron medicamento $84,4 \%$ (85 de 90) lo usaron como única alternativa terapéutica.

Con respecto a quién prescribió o recomendó el tipo de tratamiento a las 105 personas que utilizaron alguno, la mayoría refirieron que un médico recomendó el tratamiento (67 personas, 63,8\%), 7 personas siguieron recomendaciones de un profesional en farmacia, 25 personas $(23,8 \%)$ con dolor crónico se auto-medicaron, y 5 personas $(4,8 \%)$ tomaron una opción de tratamiento basados en recomendaciones de familiares, amigos 0 anuncios publicitarios. Una persona no contestó esta pregunta.

De las 90 personas que refirieron consumir medicamentos, 20 personas (18,5\%; IC95\%: 11,7-27,1) utilizaron combinación de diferentes grupos de medicamentos. Setenta y ocho personas $(72,2 \%)$ consumieron AINES, once consumieron opioides $(10,2 \%), 9$ personas $(8,3 \%)$ tomaron neuromoduladores, 9 personas $(8,3 \%)$ utilizaron vitaminas y suplementos, 6 personas $(5,6 \%)$ recurrieron a otro tipo de medicamentos como antiespasmódicos, esteroides o relajantes musculares, mientras que 6 personas acudieron a medicamentos homeopáticos. Llamó la atención que de las personas que utilizaron homeopatía, ninguna 
la utilizó en combinación con otros fármacos.

Al explorar todas las terapias no farmacológicas utilizadas (remedios caseros, fisioterapia, relajación, terapia alternativa y otros), 62 personas $(57,4 \%)$ del total de 108 con dolor crónico refirieron su uso, ya sea de forma aislada o en combinación con fármacos. De las 108 personas con dolor crónico, $33(30,6 \%)$ utilizaron remedios caseros como ungüentos hechos en casa, fórmulas con hierbas, tés, etc.; 24 (22,2\%) recurrieron a la fisioterapia, $6(5,6 \%)$ tomaron técnicas o actividades de relajación y 6 personas recurrieron a la medicina/terapia alternativa (acupuntura, iridiología, auriculoterapia, reflexología, entre otras); 9 personas $(8,3 \%)$ utilizaron otro tipo de remedios no medicamentosos no clasificados en las categorías anteriores.

En lo que respecta a la respuesta al tratamiento, el cual tiene como opciones las respuestas: Ninguna, Poca, Buena, Muy Buena, Excelente o NR, se observó que la mayoría de los entrevistados refirieron tener de poca a buena respuesta (Figura 4), lo que probablemente los conduce a tomar varios medicamentos.

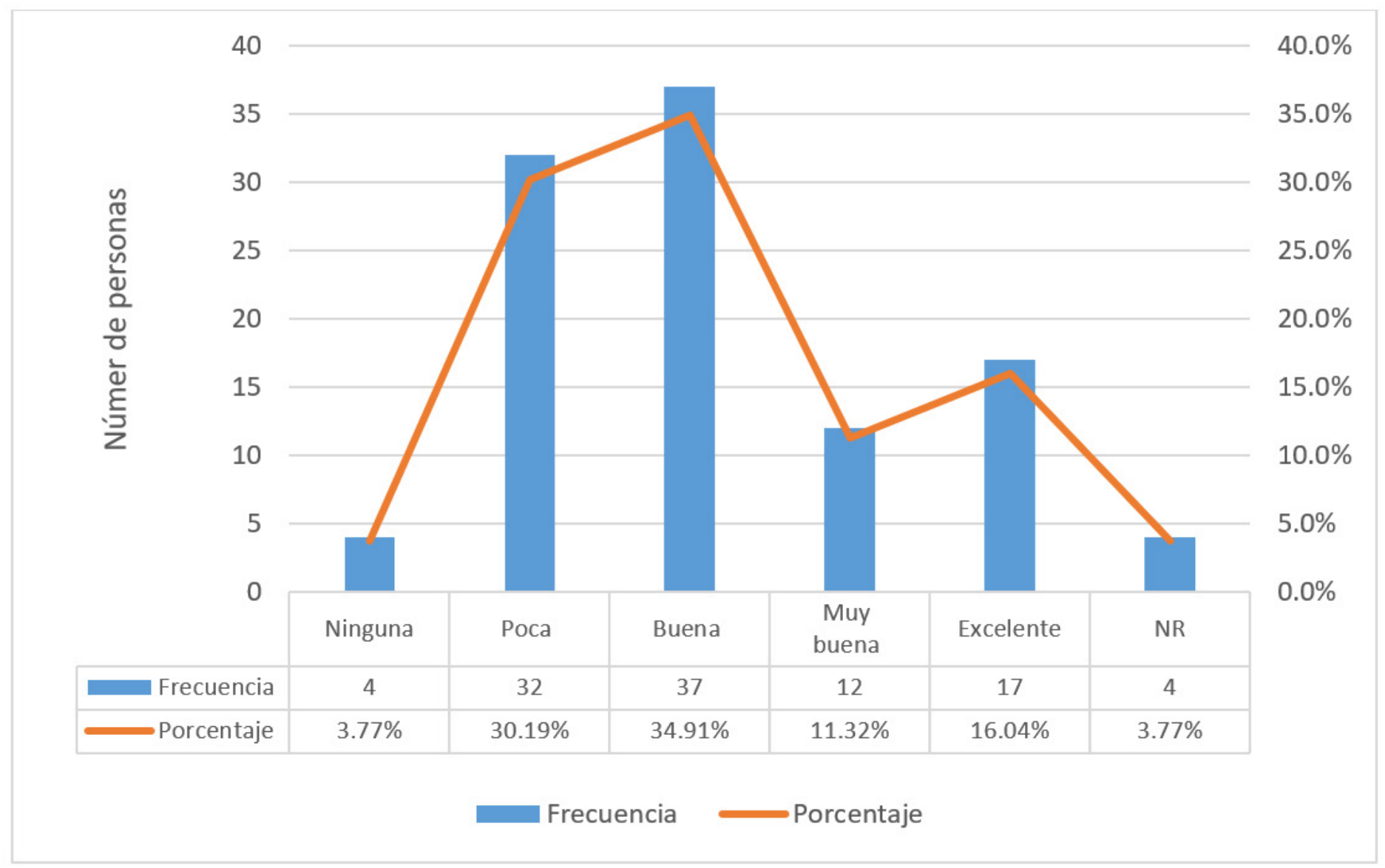

Figura 4. Distribución de personas con dolor crónico según respuesta al tratamiento, Costa Rica enero 2020- marzo 2021

Fuente: elaboración de los autores con base en datos de entrevista. 


\section{Afectación del dolor crónico en la vida de la persona}

Se preguntó a las personas con dolor crónico sobre la afectación de este en su vida. Según la escala numérica general de afectación de 1 al 10, donde 1 significa que no afecta en lo absoluto y 10 significa que le afecta enormemente, $39,8 \%$ de los participantes se ubicaron en una escala de 5 a 7 , el $29,6 \%$ se ubicaron en la escala entre $8-10$ definida como una afectación severa.

Se realizó un análisis según grado de afectación que causa el dolor crónico en los más importantes ámbitos a vida de las personas, utilizando una escala de Likert de 4 categorías: No afecta, Poco, Moderado y Severo. La Tabla 5 muestra los ámbitos y categorías incluidas, así como los resultados de este análisis.

La vida sexual, irritación y afectación emocional, marcha y el autocuido son las dimensiones menos afectadas; cerca del $30 \%$ de las personas prefirieron no contestar nada referente a la afectación de su vida sexual. La afectación en el trabajo es la más frecuente en diferentes grados de afectación (70,4\%). Ocupando el segundo puesto está la afectación de la marcha con un 65,7\%. En la categoría de afectación en el trabajo y la afectación del sueño con grado de severa son los de mayor porcentaje con un $12,0 \%$ y $15,7 \%$ respectivamente, lo que podría llevarlos a consumir medicamentos adicionales.

La prevalencia del dolor crónico en países desarrollados es del $20 \%$ y el promedio mundial es del $25 \%$ según la IASP ${ }^{8}$, nuestro estudio arrojó una prevalencia nacional de 28,8\% (IC95\%: 24,3$33,7 \%$ ) que se encuentra dentro del intervalo de confianza reportado a nivel mundial. Si se realiza la comparación con lo determinado en tres países latinoamericanos que realizaron investigaciones similares: Colombia ${ }^{12}$ reporta un $47 \%$; Brazil $^{13}$ con $39 \%$ (IC95\% 25-47\%) y Chile ${ }^{14}$ con un 32\% (IC95\%: $26.5-36 \%)$. También se encuentra Costa Rica dentro del rango de prevalencia de dolor crónico reportado por Europa en el año 2005 donde se evaluaron 15 países europeos e Israel, donde se determinó una prevalencia de dolor crónico del $12 \%$ al $30 \%{ }^{10}$. En España en el año 2015, un estudio similar al realizado en Costa Rica con 1957 encuestas telefónicas determinó que el 16,6\% tienen dolor crónico, presentándose en un porcentaje mayor en mujeres y personas adultas mayores; todos estos resultados concuerdan con nuestro estudio. Además, en dicho estudio el $12 \%$ de las personas pierden o abandonan su trabajo por esta causa ${ }^{15}$, lo que concuerda con nuestros resultados donde el área de la vida de mayor afectación fue la laboral. Según nuestros datos, el sexo femenino es el más afectado, dato que corresponde a lo encontrado en estudios realizados en Europa ${ }^{10}$, Brasil ${ }^{13}$ y Estados Unidos ${ }^{16}$.

Con respecto al tiempo de padecer de dolor crónico, $71,3 \%$ de las personas tuvieron una duración de 1 a 10 años. Se da una relación de coincidencia con el estudio europeo, donde casi el $60 \%$ tenía dolor de dos a 15 años y el $21 \%$ habían sufrido dolor durante 20 años. La mediana para los 16 países fue de 7,0 años ${ }^{10}$, comparable con la arrojada por nuestro estudio (5,16 años). Asimismo, según el estudio colombiano DOLCA ${ }^{17}$ el tiempo de los pacientes de padecer dolor crónico fue superior a un año en el $62 \%$ y de más de cinco años en el $30 \%$. 
Tabla 5. Grado de afectación de vida de las personas con dolor crónico según ámbito de la vida, Costa Rica 2020

\begin{tabular}{|c|c|c|c|c|c|c|}
\hline \multirow{2}{*}{ Ámbito de la vida } & & \multicolumn{5}{|c|}{ Grado de afectación } \\
\hline & & No afecta & Poco & Moderada & Severa & NR \\
\hline \multirow[t]{2}{*}{ Causa tristeza o depresión } & $\mathrm{n}$ & 42 & 36 & 16 & 11 & 3 \\
\hline & $\%$ & $38,9 \%$ & $33,3 \%$ & $14,8 \%$ & $10,2 \%$ & $2,8 \%$ \\
\hline \multirow[t]{2}{*}{ Afecta auto cuido } & $\mathrm{n}$ & 50 & 29 & 21 & 5 & 3 \\
\hline & $\%$ & $46,3 \%$ & $26,9 \%$ & $19,4 \%$ & $4,6 \%$ & $2,8 \%$ \\
\hline \multirow[t]{2}{*}{ Irritación y afectación emocional } & $\mathrm{n}$ & 51 & 27 & 16 & 10 & 4 \\
\hline & $\%$ & $47,2 \%$ & $25,0 \%$ & $14,8 \%$ & $9,3 \%$ & $3,7 \%$ \\
\hline \multirow[t]{2}{*}{ Afectación de la marcha } & $\mathrm{n}$ & 34 & 29 & 31 & 11 & 3 \\
\hline & $\%$ & $31,5 \%$ & $26,9 \%$ & $28,7 \%$ & $10,2 \%$ & $2,8 \%$ \\
\hline \multirow[t]{2}{*}{ Afectación vida sexual } & $\mathrm{n}$ & 55 & 6 & 12 & 3 & 32 \\
\hline & $\%$ & $50,9 \%$ & $5,6 \%$ & $11,1 \%$ & $2,8 \%$ & $29,6 \%$ \\
\hline \multirow[t]{2}{*}{ Afectación del sueño } & $\mathrm{n}$ & 39 & 33 & 14 & 17 & 5 \\
\hline & $\%$ & $36,1 \%$ & $30,6 \%$ & $13,0 \%$ & $15,7 \%$ & $4,6 \%$ \\
\hline \multirow[t]{2}{*}{ Afectación en el trabajo } & $\mathrm{n}$ & 29 & 34 & 29 & 13 & 3 \\
\hline & $\%$ & $26,9 \%$ & $31,5 \%$ & $26,9 \%$ & $12,0 \%$ & $2,8 \%$ \\
\hline \multirow[t]{2}{*}{ Afectación vida social } & $\mathrm{n}$ & 35 & 43 & 16 & 9 & 5 \\
\hline & $\%$ & $32,4 \%$ & $39,8 \%$ & $14,8 \%$ & $8,3 \%$ & $4,6 \%$ \\
\hline
\end{tabular}

Nota: la comparación porcentual es según filas para comparar los grados de afectación en cada ámbito de la vida que se ve afectado por el dolor crónico.

Fuente: elaboración de los autores con base en datos de entrevista

\section{Discusión}

Las prevalencias por provincias no presentaron diferencias estadísticamente significativas, sugiriendo que las personas con dolor crónico se distribuyen uniformemente entre las provincias de Costa Rica. La proporción de personas entrevistadas según provincia guarda relación con la densidad de población adulta de cada una de ellas. San José es la que presenta una prevalencia más cerca a la prevalencia nacional generada un $28,7 \%$, guarda relación con ser la mayor poblada de la República, más del $30 \%$ de la población costarricense habita en esta provincia, según el Instituto Nacional de Estadística y Censos $(\text { INEC })^{19}$.

Este estudio evidenció que el 31,73\% presenta más de una causa de su dolor crónico, siendo la artrosis, la lumbalgia y la fibromialgia las más frecuentes y las menos frecuentes fueron el cáncer, el herpes zoster, la artritis y los trastornos digestivos (gastritis, colitis), en comparación con el estudio europeo la Osteoartritis y artritis reumatoide combinada, fue la causa más común de dolor (42\%), solo el $1 \%$ de los encuestados 
mencionaron el cáncer, como causa de su dolor, el $12 \%$ de los encuestados no conocía la causa de su dolor ${ }^{10}$. Todas las personas entrevistadas señalaron el estrés como causa concomitante de su dolor, lo anotaron como parte de una combinación etiológica, mientras que la artrosis, etiología más frecuente del síntoma, se presentó en un $65,6 \%$ como única causa. Un estudio alemán coincide con que los factores que más frecuentemente activaron el dolor crónico fueron la tensión física en 72,6\% (65,4\%, p $<0,01)$, las condiciones climáticas en $41 \%(49,4 \%, p$ $=0,02)$ y tensión psicológica en 34,5\% $(36,9 \%)^{20}$.

En cuanto a la localización del dolor crónico se observa que el miembro inferior, seguido del miembro superior y la espalda son los sitios más frecuentes de dolor crónico, ocupando un cuarto lugar cabeza y cuello. En este sentido no se encontró una coincidencia contundente entre los estudios, pero si en las zonas del cuerpo más afectadas; el estudio colombiano ${ }^{17}$, las localizaciones más frecuentes fueron en su orden: cabeza, miembros inferiores, región lumbar, miembros superiores y abdomen. En el estudio de EEUU ${ }^{16}$ la región dorsal es el sitio más común para el dolor crónico, seguido de la rodilla y cuello.

Nuestro estudio reveló un alto porcentaje $(30 \%)$ de dolor de intensidad severa a moderada; llama la atención que en el estudio estadounidense que compara pacientes con dolor agudo y dolor crónico, estos últimos tienen más probabilidad de reportar su dolor como intenso y mayor probabilidad de acudir con frecuencia a los servicios de urgencias $^{17}$. Asimismo, en un estudio alemán ${ }^{20}$ el $73,2 \%$ de los participantes con dolor crónico estimaron entre 6 y 10 la intensidad de su dolor, en la escala de calificación numérica de 0 a 10, similar a la utilizada en este estudio.

Con respecto a la frecuencia del dolor el
$54 \%$ de la muestra de nuestro estudio presentaban el dolor a diario. Según el estudio De Andrés et $\mathrm{al}^{21}$, manifiestan que se han registrado informes de dolor crónico de un 35 a $48 \%$ de personas adultas mayores que experimentan el síntoma a diario, subiendo esta prevalencia a un $85 \%$ en las personas ingresadas en residencias.

El $2,8 \%$ no utilizan ningún tipo de tratamiento o terapia, y $88,0 \%$ utilizan un único tipo de tratamiento, el uso de medicamentos es el tratamiento más utilizado y es además el que se utiliza más como única alternativa, es decir sin combinar con otras modalidades de tratamiento como terapias, medicina alternativa o bloqueos nerviosos.

Lagran mayoríadelas personasentrevistadas con dolor crónico, un $64 \%$ refieren que un médico es el que les indica el tratamiento a seguir, sin embargo, llama la atención que cerca de una cuarta de las personas entrevistadas con dolor crónico se auto medicaron, mientras que aproximadamente $5 \%$ tomaron una opción de tratamiento basados en recomendaciones de familiares, amigos o la publicidad. En el estudio alemán se encuentra que, entre los esfuerzos propios para aliviar el dolor, la medicación analgésica fue considerada el mejor método de control manifestada por el $65 \%$ de los encuestados $^{20}$.

El grupo de medicamentos de mayor uso fueron los AINES, con frecuencia de uso significativamente superior al resto de los medicamentos. Al igual que en nuestro estudio, el estudio colombiano destaca la baja utilización de opioides y el elevado consumo de AINES, así como la habitual automedicación, todo lo cual deriva en el control inadecuado de esta patología crónica ${ }^{17}$.

En el presente estudio, 69,4\% de personas con dolor crónico manifestó afectación en las 
actividades de la vida diaria, 29,6\% la considera como una afectación severa. En comparación con el estudio de Caldas, en Colombia ${ }^{17}$ reportan que las actividades de la vida diaria (AVD) estaban limitadas de manera parcial en el $62 \%$ y total en el $13 \%$ y el dolor afectó el estado anímico en la mitad de los sujetos, se nota una diferenciación en cuanto a lo determinado en este estudio, donde el porcentaje de afectación severa a AVD es mayor y la implicación en la salud emocional es menor. Al igual que en nuestro estudio, en el estudio alemán el dolor crónico tuvo efectos negativos en el sueño en el $66,3 \%$ de los participantes y el 24,8\% necesitó usar pastillas para dormir por la afectación por el dolor ${ }^{20}$.

Las limitaciones del presente estudio son las propias de una encuesta telefónica, donde, pese al número de muestra y muestreo considerando la representatividad geográfica de la población, la mayoría de las personas que dieron su consentimiento para la entrevista fueron mujeres, donde en algunas preguntas sensibles, por ejemplo, las relacionadas con la afectación de la vida sexual, no quisieron contestar. El sesgo de memoria en temas de un padecimiento crónico puede jugar un papel importante en el estudio; así como el sesgo de información donde mucha gente puede no conocer con precisión la causa ni tratamiento de su padecimiento.

Se concluye que el dolor crónico es un problema de salud, por el número de personas que lo sufren, así como por el grado de afectación (intensidad y duración), donde la gran mayoría lo presenta de forma cotidiana y con intensidad de severa a moderada. Con la obtención de los datos epidemiológicos del dolor crónico en la población costarricense, se cuenta con información propia, que debe ser considerada en el planeamiento de terapias analgésicas y de medicamentos coadyuvantes, así como de programas en salud pública que realicen la intervención requerida en las diferentes comunidades del país.

Agradecimientos: Al Instituto de Investigación y Desarrollo Científico Académico (IIDCA) de la Universidad Santa Paula. A los encuestadores: Saindel Conejo Segura, Ariel Fernández Cordero, Josué Obando Jiménez, Fabián Mora Vega.

\section{Referencias bibliográficas}

1. International Association for the Study of Pain. Classification of Chronic Pain [Internet]. 2a ed. Seattle: IASP Press; 1994. Part III: Pain Terms, A Current List with Definitions and Notes on Usage; 209-214. Disponible en: https://s3.amazonaws.com/ rdcms-iasp/files/production/public/Content/ContentFolders/Publications2/ClassificationofChronicPain/ Part_III-PainTerms.pdf

2. López Sánchez JR, Rivera Largacha S. Historia del concepto de dolor total y reflexiones sobre la humanización de la atención a pacientes terminales. Rev Cienc Salud. 2018;16(2):340-356. doi: 10.12804/revistas.urosario.edu.co/revsalud/a.6773

3. Bilberny N. Dolor crónico en Chile. Rev Med Clin Condes. 2019;30(6):397-406. doi: 10.1016/j.rmclc.2019.08.002

4. Dorner TE. Pain and chronic pain epidemiology: Implications for clinical and public health fielDE. Wien Klin Wochenschr. 2018;130(1-2):1-3. doi: 10.1007/s00508-017-1301-0.

5. van Hecke O, Torrance N, Smith BH. Chronic pain epidemiology and its clinical relevance. $\mathrm{Br} \mathrm{J}$ Anaesth. 2013;111(1):13-8. doi: 10.1093/bja/aet123.

6. Wolkerstorfer A, Handler N, Buschmann $\mathrm{H}$. New approaches to treating pain. Bioorg Med 
Chem Lett. 2016;26(4):1103-19. doi: 10.1016/j. bmcl.2015.12.103

7. Covarrubias Gómez A, Guevara López U, Gutiérrez Salmerón C, Betancourt Sandoval JA, Córdova Domínguez JA. Epidemiología del dolor crónico en México. Rev Mex Anestesiol [Internet]. 2010;33(4):207-213. Disponible en: https://www. medigraphic.com/pdfs/rma/cma-2010/cma104e.pdf

8. Harstall C, Ospina M. How prevalent is chronic pain?. Pain Clin Updates [Internet]. 2003; 11(2): 1-4. Disponible en: https://s3.amazonaws.com/rdcms-iasp/files/production/public/Content/ContentFolders/Publications2/PainClinicalUpdates/Archives/ PCU03-2_1390265045864_38.pdf

9. Goldberg DE, McGee SJ. Pain as a global public health priority. BMC Public Health. 2011;11:770. doi: 10.1186/1471-2458-11-770.

10. Breivik H, Collett B, Ventafridda V, Cohen $\mathrm{R}$, Gallacher D. Survey of chronic pain in Europe: prevalence, impact on daily life, and treatment. Eur J Pain. 2006;10(4):287-333. doi: 10.1016/j. ejpain.2005.06.009.

11. Federación Latinoamericana de Asociaciones para el Estudio del Dolor [Internet]. San José: FEDELAT; 2018. Herramientas de Evaluación del Dolor; [aprox. 4 pantallas]. Disponible en: https:// fedelat.com/herramientas-de-evaluacion-del-dolor-2-2/

12. Guerrero Liñeiro AM, Gómez López MP. Prevalencia de dolor crónico en Colombia. VIII Estudio Nacional de Dolor [Internet]. Bogotá: ACED; 2014. Disponible en: https://www.consultorsalud.com/ wp-content/uploaDE/2014/11/viii_estudio_prevalencia_dolor_cronico_en_colombia_publicacion_pagina_aced_2014.pdf

13. de Moraes Vieira EB, Garcia JB, da Silva AA, Mualem Araújo RL, Jansen RC. Prevalence, characteristics, and factors associated with chronic pain with and without neuropathic characteristics in São Luís, Brazil. J Pain Symptom Manage. 2012;44(2):23951. doi: 10.1016/j.jpainsymman.2011.08.014.

14. Miranda JP, Morales A, Cavada G, Eberhard $\mathrm{ME}$, Ahumada M, Méndez L, et al. Validación primera encuesta chilena de Dolor Crónico no oncológico. Rev El Dolor [Internet] 2013;22(60):10-18. Disponible en: https://www.ached.cl/upfiles/revistas/documentos/54983802911a6_original1.pdf

15. Dueñas $M$, Salazar $A$, Ojeda $B$, Fernández-Palacín F, Micó JA, Torres LM, et al. A nationwide study of chronic pain prevalence in the general spanish population: identifying clinical subgroups through cluster analysis. Pain Med. 2015;16(4):81122. doi: 10.1111/pme.12640.

16. Cline DM. Dolor crónico. En: Tintinalli J, editora. Tintinalli. Medicina de urgencias. 8a ed. México: McGraw-Hill; 2018, Capítulo 38.

17. Díaz R, Marulanda F, Sáenz X. Estudio epidemiológico del dolor crónico en Caldas, Colombia (Estudio DOLCA). Acta Med Colomb. [internet] 2009;34(3):96-102. Disponible en: http://www.scielo.org.co/pdf/amc/v34n3/v34n3a2.pdf

18. Vindas Miranda TM. Respuesta del tratamiento farmacológico para el dolor crónico de tipo no oncológico en los pacientes atendidos en la clínica del dolor del Hospital Nacional Geriátrico Raúl Blanco Cervantes, durante el periodo de enero del 2014 a enero del 2015 [Tesis de Posgrado de Geriatría y Gerontología]. San José, Costa Rica: Universidad de Costa Rica; 2018. Disponible en: http://repositorio.sibdi.ucr.ac.cr:8080/jspui/bitstream/123456789/13337/1/40034.pdf

19. Instituto Nacional de Estadística y Censos (CR) [Internet]. San José: INEC; 2011. INEC publica indicadores provinciales; [aprox. 4 pantallas]. Disponible en: https://www.inec.cr/noticia/inec-publica-indicadores-provinciales 
20. Friessem $\mathrm{CH}$, Willweber-Strumpf $\mathrm{A}$, Zenz MW. Chronic pain in primary care. German figures from 1991 and 2006. BMC Public Health. 2009 18;9:299. doi: 10.1186/1471-2458-9-299.

21. De Andrés J, Acuña JP, Olivares A. Dolor en el paciente de la tercera edad. Rev Med Clin Condes. 2014;25(4):674-686. doi: 10.1016/S0716$8640(14) 70089-6$ 